\title{
Hadron Physics at KLOE and KLOE-2
}

\section{Simona GIOVANNELLA*}

Laboratori Nazionali di Frascati dell'INFN, Frascati, Italy

E-mail: simona.giovannella@lnf.infn.it

\section{on behalf of the KLOE and KLOE-2 Collaborations}

The KLOE experiment operated from 2000 to 2006 at DAФNE, the Frascati $\phi$-factory, collecting $2.5 \mathrm{fb}^{-1}$ of $e^{+} e^{-}$collisions at the $\phi$ peak and $240 \mathrm{pb}^{-1}$ at $\sqrt{s}=1 \mathrm{GeV}$. Data taken on peak have been used for several measurements on light meson spectroscopy. With the off-peak data, studies of the reactions $e^{+} e^{-} \rightarrow e^{+} e^{-} \eta$ and $e^{+} e^{-} \rightarrow e^{+} e^{-} \pi^{0} \pi^{0}$ have been performed. Most recent results and perpectives for the KLOE-2 run, where a larger data sample is expected, will be discussed.

8th International Conference on Nuclear Physics at Storage Rings-Stori11,

October 9-14, 2011

Laboratori Nazionali di Frascati dell'INFN, Italy

\footnotetext{
* Speaker.
} 


\section{Introduction}

From 2000 to 2006, the KLOE experiment collected $2.5 \mathrm{fb}^{-1}$ of $e^{+} e^{-}$collisions at the $\phi$ peak and $240 \mathrm{pb}^{-1}$ below the $\phi$ resonance $(\sqrt{s}=1 \mathrm{GeV})$. The $\phi$ meson predominantly decays into charged and neutral kaons, thus allowing KLOE to make precision studies in the fields of flavor physics, low energy QCD and test of discrete symmetries. At the same time, properties of pseudoscalar and scalar mesons have been extensively studied using high statistic samples produced through $\phi$ radiative decays and $\gamma \gamma$ interactions.

A new beam crossing scheme allowing for a reduced beam size and increased luminosity is operating at DAФNE [1]. The KLOE-2 detector is successfully rolled in and has been upgraded with small angle tagging devices to detect both high and low energy electrons or positrons in $e^{+} e^{-} \rightarrow e^{+} e^{-} X$ events. About $5 \mathrm{fb}^{-1}$ are expected in the first year of running. Inner tracker and small angle calorimeters are scheduled to be installed in a subsequent step, providing larger acceptance both for charged particles and photons. A detailed description of the KLOE-2 physics program can be found in Ref. [2]

\section{The $\phi \rightarrow \eta e^{+} e^{-}$decay}

The $V \rightarrow P \gamma^{*}$ Dalitz decays, associated to internal conversion of the photon into a lepton pair, are not well described by the Vector Meson Dominance (VMD) model. This is clearly visible in the di-lepton invariant mass shape of $\omega \rightarrow \pi^{0} \ell^{+} \ell^{-}$, measured by the NA60 collaboration [3]. The only existing data on $\phi \rightarrow \eta e^{+} e^{-}$come from the SND experiment, which has measured the $\mathrm{M}_{e e}$ invariant mass distribution on the basis of 213 events [4]. Also in this case, the measurement of the form factor slope differs by $\sim 2 \sigma$ 's from the VMD expectations. A new measurement of this process with larger statistics is therefore needed to confirm this indication. This decay is also interesting for the search of a possible contribution from $\phi \rightarrow \eta U$, where $U$ is a vector gauge boson, with mass near the GeV scale, coupling the postulated secluded sector of WIMP dark matter to the Standard Model $[5,6,7,8,9,10,11,12,13,14]$. The kinetic mixing parameter, $\varepsilon$, is expected to be of the order $10^{-4}-10^{-2}[6,15]$.

The analysis of the $\phi \rightarrow \eta e^{+} e^{-}$, with $\eta \rightarrow \pi^{+} \pi^{-} \pi^{0}$ has been performed on $1.5 \mathrm{fb}^{-1}$. Preselection cuts require: (i) four tracks in a cylinder around the interaction point (IP) plus two photon candidates; (ii) best $\pi^{+} \pi^{-} \gamma \gamma$ match to the $\eta$ mass using the pion hypothesis for tracks; (iii) other two tracks assigned to $e^{+} e^{-}$; (iv) loose cuts on $\eta$ and $\pi^{0}$ invariant masses $\left(495<M_{\pi^{+}} \pi^{-} \gamma \gamma<600\right.$ $\left.\mathrm{MeV}, 70<M_{\gamma \gamma}<200 \mathrm{MeV}\right)$. A cut in the distribution of the recoil mass to the $e^{+} e^{-}$pair, $535<$ $M_{\text {recoil }}(e e)<560 \mathrm{MeV}$ is then applied.

A residual background contamination, due to $\phi \rightarrow \eta \gamma$ events with photon conversion on beam pipe (BP) or drift chamber walls (DCW), is rejected by tracking back to BP/DCW surfaces the two $e^{+}, e^{-}$candidates and then reconstructing the electron-positron invariant mass and the distance between the two particles. Both quantities are small if coming from photon conversion. $\phi \rightarrow$ $K \bar{K}$ and $\phi \rightarrow \pi^{+} \pi^{-} \pi^{0}$ events surviving analysis cuts have more than two pions in the final state. They are rejected using time-of-flight to the calorimeter. When an EMC cluster is connected to a track, the arrival time to the calorimeter is evaluated both with calorimeter $\left(T_{\text {cluster }}\right)$ and drift 

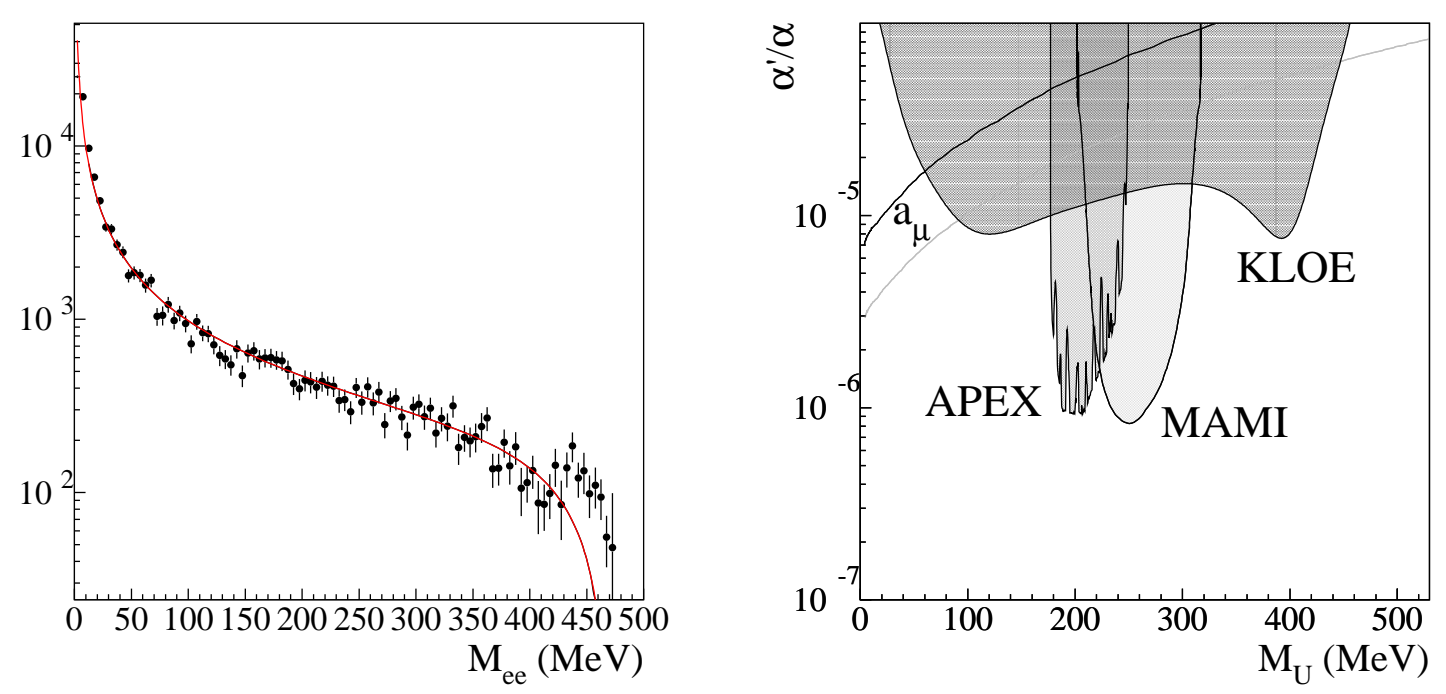

Figure 1: Fit to the corrected $M_{e e}$ spectrum for the Dalitz decay $\phi \rightarrow \eta e^{+} e^{-}$(left) and exclusion plot at $90 \%$ C.L. for the parameter $\alpha^{\prime} / \alpha=\varepsilon^{2}$, compared with existing limits in the KLOE region of interest (right).

chamber $\left(T_{\text {track }}\right)$ information. Events with an $e^{+}, e^{-}$candidate outside a $3 \sigma$ 's window on the $D T=T_{\text {track }}-T_{\text {cluster }}$ variable are rejected.

About $14,000 \phi \rightarrow \eta e^{+} e^{-}, \eta \rightarrow \pi^{+} \pi^{-} \pi^{0}$ candidates are present in the analyzed data set, with a very small residual background contamination. The resulting electron-positron invariant mass shape, $M_{e e}$, has been fitted, taking into account bin-by-bin analysis efficiency and using the decay parametrization from Ref. [16]. Fit results are reported in Fig. 1 left. A good description of the $M_{e e}$ shape is obtained except at the high end of the spectrum, where a residual background contamination from multi-pion events is still present.

The $\phi \rightarrow \eta U$ Monte Carlo (MC) signal has been produced according to Ref. [17], with a flat distribution of the $U$ boson mass. Events are then divided in sub-samples of $1 \mathrm{MeV}$ width. For each $M_{e e}$ value, signal hypothesis has been excluded at $90 \%$ C.L. using the $\mathrm{CL}_{\mathrm{S}}$ technique [18]. For the $\phi \rightarrow \eta U$ signal, the opening of the $U \rightarrow \mu^{+} \mu^{-}$threshold has been included, in the hypothesis that the $U$ boson decays only to lepton pairs and assuming equal coupling to $e^{+} e^{-}$and $\mu^{+} \mu^{-}$. The expected shape for the irreducible background $\phi \rightarrow \eta e^{+} e^{-}$is obtained from our fit to the $M_{e e}$ distribution, taking also into account the error on the number of background events as a function of $M_{e e}$. In Fig. 1 right, the smoothed exclusion plot at 90\% C.L. on $\alpha^{\prime} / \alpha=\varepsilon^{2}$ parameter is compared with existing limits from the muon anomalous magnetic moment $a_{\mu}$ [19] and from recent measurements of the MAMI/A1 [20] and APEX [21] experiments. The gray line is where the $U$ boson parameters should lay to account for the observed discrepancy between measured and calculated $a_{\mu}$ values. Our result greatly improves existing limits in a wide mass range, resulting in an upper limit on the $\alpha^{\prime} / \alpha$ parameter of $\leq 2 \times 10^{-5} @ 90 \%$ C.L. for $50<M_{U}<420 \mathrm{MeV}$.

\section{The $\eta \rightarrow \pi^{+} \pi^{-} \gamma$ decay}

The $\eta \rightarrow \gamma \gamma$ decay proceeds through the triangle anomaly and is accounted for by the Wess- 

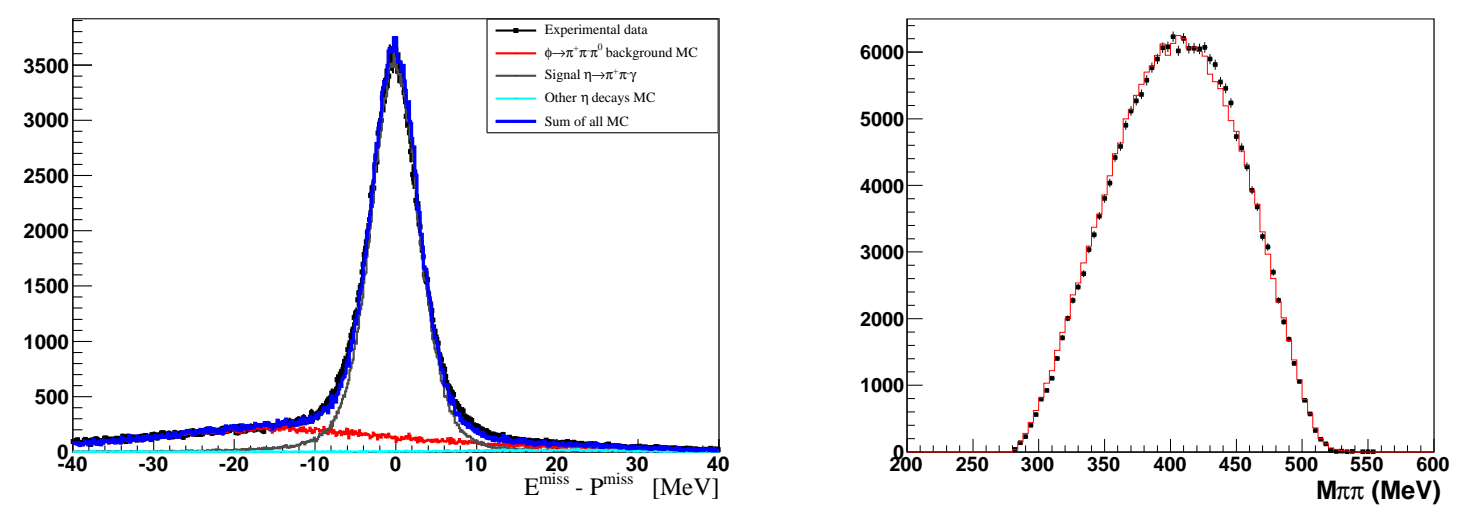

Figure 2: $\eta \rightarrow \pi^{+} \pi^{-} \gamma$ analysis: fit to the $E_{\mathrm{miss}}-P_{\mathrm{miss}}$ distribution with background and signal MC shapes (left) and fit to the $\pi^{+} \pi^{-}$invariant mass (right).

Zumino-Witten (WZW) term into the ChPT Lagrangian. The box anomaly is a higher term of WZW describing the direct coupling of three pseudoscalar mesons with the photon. The $\eta \rightarrow$ $\pi^{+} \pi^{-} \gamma$ decay provides a good tool to investigate the box anomaly, which describes the notresonant part of the coupling, through the measurement of the partial width and the study of the invariant mass of the di-pion system. The present world average of the $\eta \rightarrow \pi^{+} \pi^{-} \gamma$ partial width, $\Gamma\left(\eta \rightarrow \pi^{+} \pi^{-} \gamma\right)=(60 \pm 4) \mathrm{eV}$ [23], provides strong evidence in favor of the box anomaly, compared with value expected with and without the direct term [22]: $(56.3 \pm 1.7) \mathrm{eV}$ and $(100.9 \pm 2.8) \mathrm{eV}$, respectively. Recently the CLEO experiment has measured the ratio $R_{\eta}=$ $\Gamma\left(\eta \rightarrow \pi^{+} \pi^{-} \gamma\right) / \Gamma\left(\eta \rightarrow \pi^{+} \pi^{-} \pi^{0}\right)=0.175 \pm 0.007_{\text {stat }} \pm 0.006_{\text {syst }}$ [26], which differs by more than $3 \sigma$ 's from the average result of previous measurements [24, 25], $R_{\eta}=0.207 \pm 0.004$.

The analysis of $\eta \rightarrow \pi^{+} \pi^{-} \gamma$ has been performed using $558 \mathrm{pb}^{-1}$, where about $25 \times 10^{6} \eta$ 's are produced together with a monochromatic recoil photon $\left(\gamma_{\phi}\right.$, with $\left.E_{\gamma_{\phi}}=363 \mathrm{MeV}\right)$ through the radiative decay $\phi \rightarrow \eta \gamma$. The final state under study is therefore $\pi^{+} \pi^{-} \gamma \gamma$ and the main background contribution comes from $\phi \rightarrow \pi^{+} \pi^{-} \pi^{0}$ events, with the same final state. Other relevant background components are $\phi \rightarrow \eta \gamma \rightarrow \pi^{+} \pi^{-} \pi^{0} \gamma$, with one photon lost, and $\phi \rightarrow \eta \gamma \rightarrow e^{+} e^{-} \gamma \gamma$, with both electrons mis-identified as pions. After preselection cuts used to clean up the data sample, signal events are selected exploiting the $\phi \rightarrow \eta \gamma$ two body decay kinematics. Background contribution and the signal amount in the final sample are evaluated with a fit to the $E_{\text {miss }}-P_{\text {miss }}$ distribution with MC shapes (Fig. 2 left), where $E_{\text {miss }}$ and $P_{\text {miss }}$ are the missing energy and momentum of the $\pi^{+} \pi^{-} \gamma_{\phi}$ system, respectively. We find $N\left(\eta \rightarrow \pi^{+} \pi^{-} \gamma\right)=204950 \pm 450$, with a $10 \%$ background contamination. The process $\phi \rightarrow \eta \gamma, \eta \rightarrow \pi^{+} \pi^{-} \pi^{0}$, with similar event topology and negligible background contamination, has been used as normalization sample. The preliminary ratio of the partial decay widths is:

$$
\frac{\Gamma\left(\eta \rightarrow \pi^{+} \pi^{-} \gamma\right)}{\Gamma\left(\eta \rightarrow \pi^{+} \pi^{-} \pi^{0}\right)}=0.1838 \pm 0.0005_{\text {stat }} \pm 0.0030_{\text {syst }},
$$

to be compared with the world average value of $0.202 \pm 0.007$ [23].

The $M_{\pi^{+} \pi^{-}}$dependence of the $\eta \rightarrow \pi^{+} \pi^{-} \gamma$ decay width has been parameterized with several theoretical approaches. In Fig. 2 right, the preliminary fit of the background subtracted di-pion 


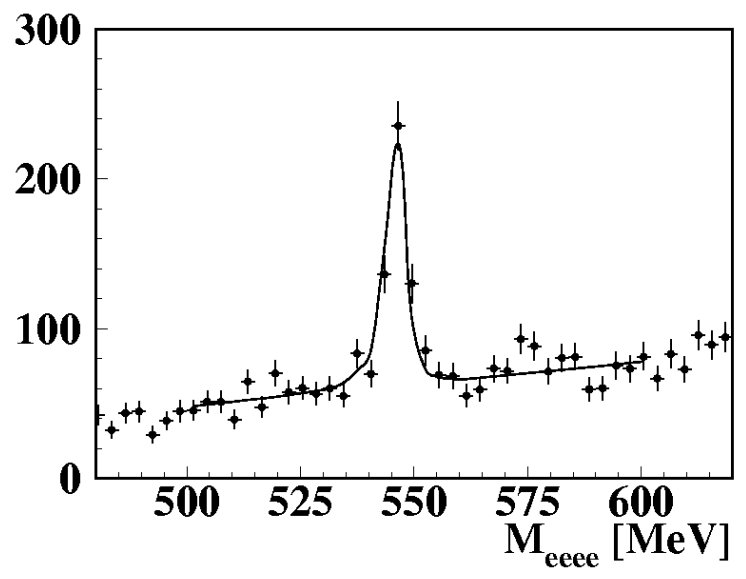

Figure 3: Fit to the $e^{+} e^{-} e^{+} e^{-}$invariant mass used to extract the $\eta \rightarrow e^{+} e^{-} e^{+} e^{-}$contribution.

invariant mass spectrum, using the description of Ref. [27], is presented. The agreement is good. Fits with more complex parameterizations are in progress.

\section{Observation of the $\eta \rightarrow e^{+} e^{-} e^{+} e^{-}$decay}

The search of the rare and never observed decay $\eta \rightarrow e^{+} e^{-} e^{+} e^{-}$has been performed using 1.7 $\mathrm{fb}^{-1}$. Events with four $e^{ \pm}$in the final state are selected using the time of flight information provided by the calorimeter. Backgrounds from $\eta \rightarrow \gamma \gamma / e^{+} e^{-} \gamma$ with photon conversion are rejected by reconstructing the conversion point on the beam pipe or drift chamber walls. Most of the residual background comes from continuum events and a small contribution is due to $\phi$ decays. The latter is subtracted from data using the MC spectrum. The number of signal events is obtained by fitting the data distribution of the four lepton invariant mass, $\mathrm{M}_{\text {eeee }}$, with signal and background shapes (Fig. 3). The resulting $\chi^{2} /$ ndf is $43.9 / 34$, corresponding to $\mathrm{P}\left(\chi^{2}\right)=0.12$. From the fit we extract $N_{\eta \rightarrow e^{+} e^{-} e^{+} e^{-}}=362 \pm 29$, corresponding to a branching fraction of [28]

$$
\mathrm{BR}\left(\eta \rightarrow e^{+} e^{-} e^{+} e^{-}(\gamma)\right)=\left(2.4 \pm 0.2_{\text {stat }+ \text { bckg }} \pm 0.1_{\text {syst }}\right) \times 10^{-5},
$$

fully inclusive of radiation effects. This constitutes the first observation of this decay. Our measurement is in agreement with theoretical predictions, which are in the range $(2.41-2.67) \times 10^{-5}$ $[29,30,31]$.

\section{5. $\gamma \gamma$-physics}

The study of the reaction $e^{+} e^{-} \rightarrow e^{+} e^{-} \gamma^{*} \gamma^{*} \rightarrow e^{+} e^{-} X$, where $X$ is some arbitrary final state resulting from fusion of two photons, gives access to states with $J^{P C}=0^{ \pm+}, 2^{ \pm+}$. In the low-energy region accessible at DA $\Phi$ NE, several existing measurements are affected by large statistical and systematic uncertainties. At KLOE, where there is no tagging of the outgoing $e^{+} e^{-}, \gamma \gamma$ interactions have been studied using off-peak data $\left(240 \mathrm{pb}^{-1}\right.$ collected at $\left.\sqrt{s}=1 \mathrm{GeV}\right)$, as the main source of background comes from $\phi$ decays. 

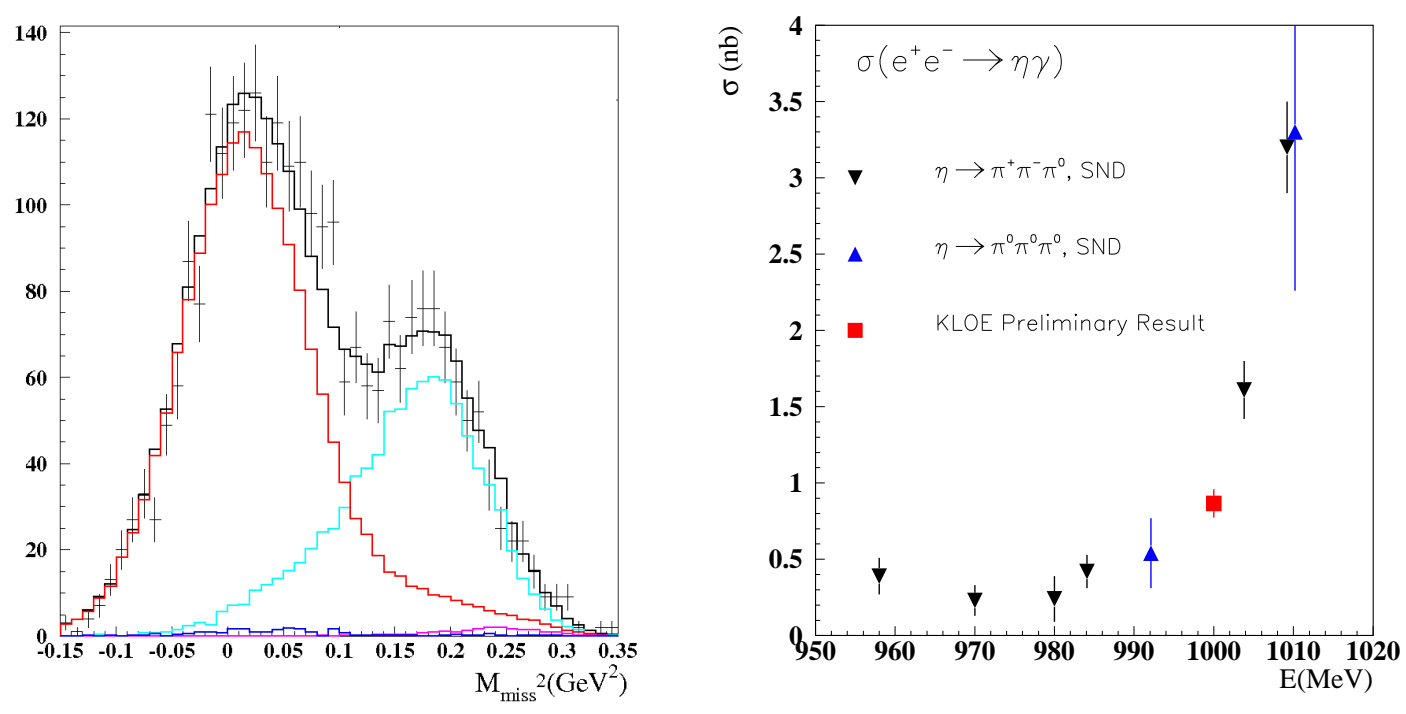

Figure 4: Left: Fit to the $M_{\text {miss }}^{2}$ distribution for $\gamma \gamma \rightarrow \eta \rightarrow \pi^{0} \pi^{0} \pi^{0}$ events. Points with error bars are data, black solid histogram is fit result. The main components are signal (light blue) and $\phi \rightarrow \eta \gamma$ (red.) Right: preliminary KLOE measurement for $\sigma_{e^{+} e^{-} \rightarrow \eta \gamma}$ evaluated at $1 \mathrm{GeV}$, compared with other experimental results in the same energy range.

The $\eta$ radiative width, $\Gamma(\eta \rightarrow \gamma \gamma)$, is usually extracted from the measurement of the $e^{+} e^{-} \rightarrow$ $e^{+} e^{-} \eta$ cross section. The KLOE analysis is performed using both neutral $\left(\eta \rightarrow \pi^{0} \pi^{0} \pi^{0}\right)$ and charged ( $\eta \rightarrow \pi^{+} \pi^{-} \pi^{0}$ ) decay channels. The main backgrounds for $\eta \rightarrow \pi^{0} \pi^{0} \pi^{0}$ are annihilation processes with at least four prompt photons in the final state, as $e^{+} e^{-} \rightarrow \eta \gamma / K_{S} K_{L} / \omega \pi^{0}$. Events with six and only six prompt photons in the final state and with no tracks in the drift chamber are selected. The photons are paired choosing the combination which minimizes a $\chi^{2}$-like variable based on the $\pi^{0}$ masses. A kinematic fit is then performed asking for the six photons invariant mass to be equal to the mass of the $\eta$ meson. A cut is then applied on the energy of the most energetic photon to reject $e^{+} e^{-} \rightarrow \eta \gamma$ events in which the monochromatic photon $\left(E_{\gamma}=350 \mathrm{MeV}\right)$ is detected. The only surviving background events are due to the irreducible process $e^{+} e^{-} \rightarrow \eta \gamma$, with the monochromatic photon lost in the beam pipe. The distribution of the recoil missing mass $\left(M_{m i s s}^{2}\right)$ is fitted with the superposition of MC shapes for signal and background (Fig. 4 left). From this fit we extract the preliminary measurement of both the $\eta$ production cross section, $\sigma(\gamma \gamma \rightarrow$ $\eta)=\left(37.0 \pm 1.4_{\text {stat }} \pm 2.2_{\text {syst }}\right) \mathrm{pb}$, and $\sigma\left(e^{+} e^{-} \rightarrow \eta \gamma, \sqrt{s}=1 \mathrm{GeV}\right)=\left(0.875 \pm 18_{\text {stat }} \pm 35_{\text {syst }}\right) \mathrm{nb}$. This last result is shown in Fig. 4 right, together with other experimental results [32] in the same energy range. The selection of $\eta \rightarrow \pi^{+} \pi^{-} \pi^{0}$ asks for two photons compatible with a $\pi^{0}$ decay and two tracks with opposite curvature coming from the collision point. The charged pion mass is assigned to the two tracks and a least squares function based on Lagrange multipliers imposes that $\pi^{+} \pi^{-} \pi^{0}$ come from an $\eta$ decay. Further criteria are applied to suppress processes with photons and $e^{+} e^{-}$in the final state. The distribution of $M_{m i s s}^{2}$ is then fitted with the superposition of MC shapes for signal and background. The preliminary measurements of the $\eta$ production cross section 

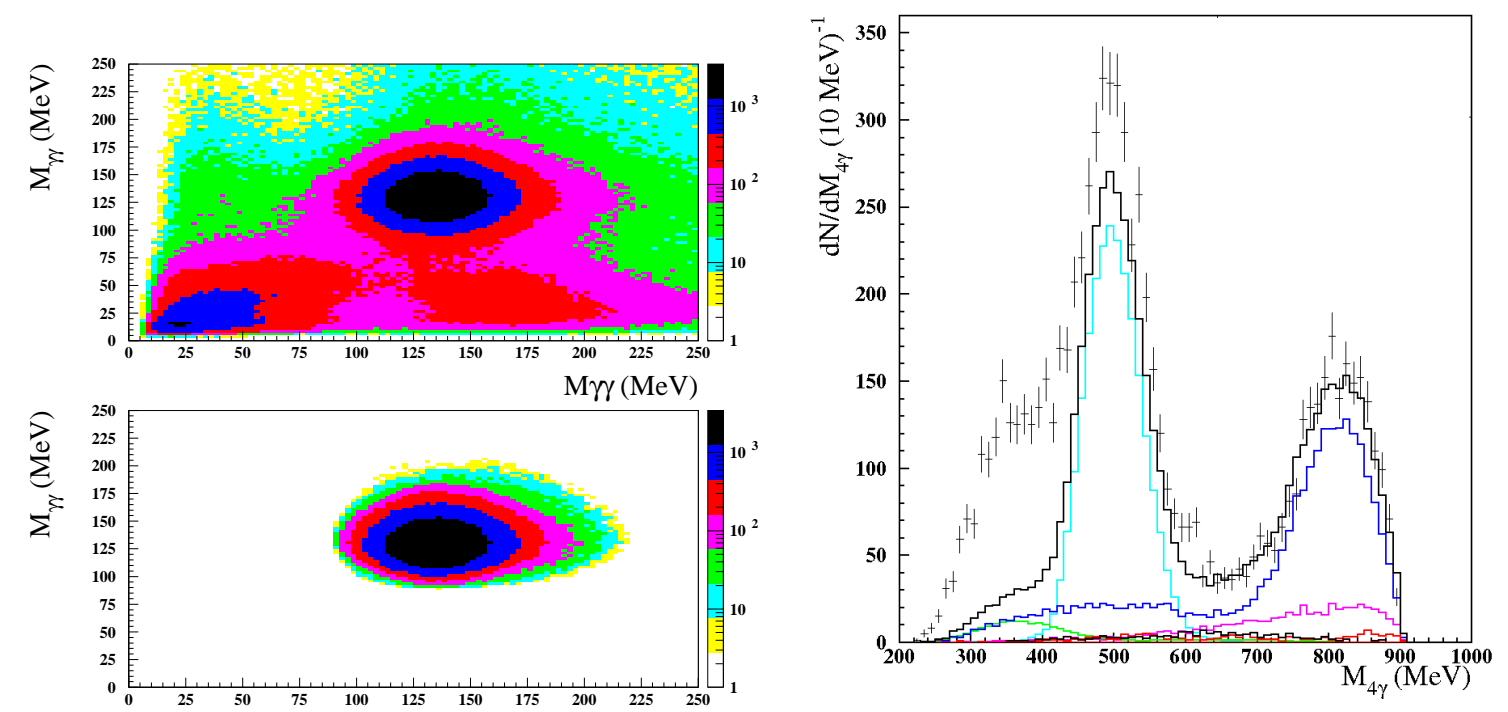

Figure 5: $\gamma \gamma \rightarrow \pi^{0} \pi^{0}$ analysis. Left: scatter plots of the two photon pairs invariant mass before (top) and after (bottom) the cut on the $\chi_{4 \gamma}^{2}$ variable. Right: spectrum of the four-photon invariant mass (points with error bars) compared with the sum of normalized backgrounds (black solid line). The main background contributions are shown in colours: $\phi \rightarrow K_{S} K_{L}$ (light blue), $e^{+} e^{-} \rightarrow \omega \pi^{0}$ (blue), $\phi \rightarrow f_{0}(980) \gamma$ (pink), $\phi \rightarrow \eta \gamma$ (green) and $e^{+} e^{-} \rightarrow \gamma \gamma$ (red).

is $\sigma(\gamma \gamma \rightarrow \eta)=\left(41.7 \pm 4.0_{\text {stat }}\right) \mathrm{pb}$, in agreement with the measurement obtained with the neutral channel. Systematics are still under evaluation.

The study of the $e^{+} e^{-} \rightarrow e^{+} e^{-} \pi^{0} \pi^{0}$ process aims to investigate the low $\pi^{0} \pi^{0}$ invariant mass region, just above the production threshold, where a contribution from the $\sigma$ scalar meson is expected. The main backgrounds are annihilation processes with four or more prompt photons in the final state $\left(e^{+} e^{-} \rightarrow K_{S} K_{L} / \eta \gamma / \omega \pi^{0} / f_{0}(980) \gamma\right)$ and the $e^{+} e^{-} \rightarrow \gamma \gamma$ process with cluster splitting. Selected events have no tracks in the drift chamber and four prompt photons in the final state. The photons are paired choosing the combination which minimizes a $\chi^{2}$-like variable based on $\pi^{0}$ masses, $\chi_{4 \gamma}^{2}$. Events with $\chi_{4 \gamma}^{2}>4$ are rejected. The effect of this selection is shown in Fig. 5 left. To reject $e^{+} e^{-} \rightarrow K_{S} K_{L}$ events, where a large amount of non-prompt energy is released in the detector, a cut on the energy fraction carried by photons, $R=\left(\sum_{\gamma} E_{\gamma}\right) / E_{c a l}>0.75$, is applied. The preliminary four photon invariant mass spectrum for the selected data sample is shown in Fig. 5 right, together with the normalized background Monte Carlo simulations. The excess of events with respect to the expected annihilation processes, in the low invariant mass region, is an indication of the $\gamma \gamma \rightarrow \pi^{0} \pi^{0}$ production.

The upgrade of the KLOE- 2 detector, with four stations installed to tag electrons and positrons from the reaction $e^{+} e^{-} \rightarrow e^{+} e^{-} \gamma^{*} \gamma^{*} \rightarrow e^{+} e^{-} X$, will give the opportunity to investigate $\gamma \gamma$ physics also at the $\phi$ resonance for the reactions $\gamma \gamma \rightarrow \pi^{0} / \pi \pi / \eta / \eta \pi$ [2] . Single pseudoscalar production would improve the determination of the two-photon decay widths of these mesons. Moreover, it would be possible to measure the transition form factors $\mathscr{F}_{X \gamma^{*} \gamma^{*}}\left(q_{1}^{2}, q_{2}^{2}\right)$ as a function of the 
momentum of the virtual photons, $q_{1}^{2}$ and $q_{2}^{2}$, which are of interest for the theoretical evaluation of the hadronic light-by-light contribution to the muon magnetic anomaly.

\section{References}

[1] C. Milardi et al., ICFA Beam Dyn. Newslett. 48, 23 (2009).

[2] G. Amelino-Camelia et al., Eur. Phys. J. C 68, 619 (2010).

[3] S. Damjanovic et al., Phys. Lett. B 677, 260 (2009).

[4] M. N. Achasov et al., Phys. Lett. B 504, 275 (2001).

[5] M. Pospelov, A. Ritz, M.B. Voloshin, Phys. Lett. B 662, 53 (2008).

[6] N. Arkani-Hamed, D.P. Finkbeiner, T.R. Slatyer, N. Weiner, Phys. Rev. D 79, 015014 (2009).

[7] D.S.M. Alves, S.R. Behbahani, P. Schuster, J.G. Wacker, Phys. Lett. B 692, 323 (2010).

[8] M. Pospelov, A. Ritz, Phys. Lett. B 671, 391 (2009).

[9] J. Hisano, S. Matsumoto, M.M. Nojiri, Phys. Rev. Lett. 92, 031303 (2004).

[10] M. Cirelli, M. Kadastik, M. Raidal, A. Strumia, Nucl. Phys. B 813, 1 (2009).

[11] J. March-Russell, S.M. West, D. Cumberbatch, D. Hooper, JHEP 07, 058 (2008).

[12] I. Cholis, G. Dobler, D.P. Finkbeiner, L. Goodenough, N. Weiner, Phys. Rev. D 80, 123518 (2009).

[13] I. Cholis, D.P. Finkbeiner, L. Goodenough, N. Weiner, JCAP 0912, 007 (2009).

[14] N. Arkani-Hamed, N. Weiner, JHEP 12, 104 (2008).

[15] R. Essig, P. Schuster, N. Toro, Phys. Rev. D 80, 015003 (2009).

[16] L.G. Landsberg, Phys. Rep. 128, 301 (1985).

[17] M. Reece, L.T. Wang, JHEP 07, 051 (2009).

[18] T. Junk, Nucl. Instr. Meth. A 434, 435 (1999).

[19] M. Pospelov, Phys. Rev. D 80, 095002 (2009).

[20] H. Merkel et al., Phys. Rev. Lett. 106, 251802 (2011).

[21] S. Abrahamyan et al., Phys. Rev. Lett. 107, 191804 (2011).

[22] M. Benayoun et al., Eur. Phys. J. C 31, 525 (2003).

[23] K. Nakamura et al., J. Phys. G 37, 075021 (2010).

[24] P. Gormley, Phys. Rev. D 2, 501 (1970).

[25] J. Layter, Phys. Rev. D 7, 2565 (1973).

[26] A. Lopez et al., Phys. Rev. Lett. 99, 122001 (2007).

[27] C. Picciotto, Phys. Rev. D 45, 1569 (1992).

[28] F. Ambrosino et al., Phys. Lett. B 702, 324 (2011).

[29] C. Jarlskog and H. Pilkuhn, Nucl. Phys. B 1, 264 (1967).

[30] T. Miyazaki and E. Takasugi, Phys. Rev. D 8, 2051 (1973).

[31] C. C. Lih, J. Phys. G 38, 065001 (2011).

[32] M. N. Achasov et al., Phys. Rev. D 76, 077101 (2007). 